\title{
A Result on the Existence and Uniqueness of Stationary Solutions for a Bioconvective Flow Model
}

\author{
Aníbal Coronel (D), Luis Friz, Ian Hess, and Alex Tello \\ GMA, Departamento de Ciencias Básicas, Facultad de Ciencias, Universidad del Bío-Bío, Campus Fernando May, Chillán, Chile
}

Correspondence should be addressed to Aníbal Coronel; acoronel@ubiobio.cl

Received 8 December 2017; Accepted 25 March 2018; Published 30 April 2018

Academic Editor: Juan Martinez-Moreno

Copyright (C) 2018 Aníbal Coronel et al. This is an open access article distributed under the Creative Commons Attribution License, which permits unrestricted use, distribution, and reproduction in any medium, provided the original work is properly cited.

In this note, we prove the existence and uniqueness of weak solutions for the boundary value problem modelling the stationary case of the bioconvective flow problem. The bioconvective model is a boundary value problem for a system of four equations: the nonlinear Stokes equation, the incompressibility equation, and two transport equations. The unknowns of the model are the velocity of the fluid, the pressure of the fluid, the local concentration of microorganisms, and the oxygen concentration. We derive some appropriate a priori estimates for the weak solution, which implies the existence, by application of Gossez theorem, and the uniqueness by standard methodology of comparison of two arbitrary solutions.

\section{Introduction}

Bioconvection is an important process in the biological treatment and in the life of some microorganisms. In a broad sense, biconvection originates from the concentration of upward swimming microorganisms in a culture fluid. It is well known that, under some physical assumptions, the process can be described by mathematical models which are called bioconvective flow models. The first model of this kind was derived by Moribe [1] and independently by Levandowsky et al. [2] (see also [3] for the mathematical analysis). In that model the unknowns are the velocity of the fluid, the pressure of the fluid, and the local concentration of microorganisms. More recently, Tuval et al. [4] have introduced a new bioconvective flow model considering an additional unknown variable, the oxygen concentration. Some advances in mathematical analysis and some numerical results for this new model are presented in [5] and [6], respectively.

In this paper, we are interested in the existence and uniqueness of solutions for the stationary problem associated with the bioconvective system given in [4] when the physical domain is a three-dimensional chamber [6] (a parallelepiped). Thus, the stationary bioconvective flow problem to be analyzed is formulated as follows. Given the external force $\mathbf{F}$, the source functions $f_{n}, f_{c}$, and the dimensionless function $r$, find the velocity of the fluid $\mathbf{u}=\left(u_{1}, u_{2}, u_{3}\right)^{t}$, the fluid pressure $p$, the local concentration of bacteria $n$, and the local concentration of oxygen $c$ satisfying the boundary value problem:

$$
\begin{aligned}
&-S_{c} \Delta \mathbf{u}+(\mathbf{u} \cdot \nabla) \mathbf{u}+S_{c} \nabla p=\gamma S_{c} n \mathbf{g}+\mathbf{F}, \\
& \text { in } \Omega:=\prod_{i=1}^{3}\left[0, L_{i}\right], \\
& \operatorname{div}(\mathbf{u})=0, \quad \text { in } \Omega, \\
&-\Delta n+(\mathbf{u} \cdot \nabla) n+\chi \operatorname{div}(n r(c) \nabla c)=f_{n}, \quad \text { in } \Omega, \\
&-\delta \Delta c+(\mathbf{u} \cdot \nabla) c+\beta r(c) n=f_{c}, \quad \text { in } \Omega, \\
& \nabla c \cdot \boldsymbol{v}=\nabla n \cdot \boldsymbol{v}=0, \\
& \mathbf{u}=0, \\
& \text { on } \partial \Omega_{L}\left(x_{3}=0\right), \\
& \chi n r(c) \nabla c \cdot v-\nabla n \cdot \boldsymbol{v}=0, \\
& \mathbf{u}=0, \\
& \text { on } \partial \Omega_{U}:=\partial \Omega-\partial \Omega_{L} .
\end{aligned}
$$


Here $\boldsymbol{v}$ is the unit external normal to $\partial \Omega ; \mathbf{g}=(0,0,-g)$ is a gravitational field with constant acceleration $g$; and $S_{c}, \gamma, \alpha, \delta$, and $\beta$ are some physical parameters defined as follows:

$$
\begin{aligned}
S_{c} & =\frac{\eta}{D_{n} \rho}, \\
\gamma & =\frac{V_{b} n_{r}\left(\rho_{b}-\rho\right) L^{3}}{\eta D_{n}}, \\
\chi & =\frac{\bar{\chi} c_{\text {air }}}{D_{n}}, \\
\delta & =\frac{D_{c}}{D_{n}}, \\
\beta & =\frac{k n_{r} L^{2}}{c_{\text {air }} D_{n}},
\end{aligned}
$$

with $\eta$ being the fluid viscosity, $D_{n}$ the diffusion constant for bacteria, $D_{c}$ the diffusion constant for oxygen, $\rho$ the fluid density, $\rho_{b}$ the bacterial density, $V_{b}>0$ the bacterial volume, $n_{r}$ a characteristic cell density, $L$ a characteristic length, $\bar{\chi}$ the chemotactic sensitivity, $c_{\text {air }}$ the oxygen concentration above the fluid, and $k$ the oxygen consumption rate.

We consider the standard notation of the Lebesgue and Sobolev spaces which are used in the analysis of NavierStokes and related equations of fluid mechanics; see [7-11] for details and specific definitions. In particular, we use the following rather common spaces notation:

$$
\begin{aligned}
H^{m}(\Omega) & =W^{m, 2}(\Omega), \\
\widetilde{H}^{1}(\Omega) & =\left\{f \in H^{1}(\Omega): \int_{\Omega} f d \mathbf{x}=0\right\}, \\
H_{0}^{1}(\Omega) & =\overline{\mathbf{C}}_{0}^{\infty}(\Omega) \\
\mathbf{C}_{0, \sigma}^{\infty}(\Omega) \|_{H^{1}(\Omega)}, & =\left\{\mathbf{v} \in\left(C_{0}^{\infty}(\Omega)\right)^{3}: \operatorname{div}(\mathbf{v})=0\right\}, \\
\mathbf{V} & =\overline{\mathbf{C}}_{0, \sigma}^{\infty}(\Omega)^{\|\cdot\|_{H_{0}^{1}(\Omega)}},
\end{aligned}
$$

where $\bar{A}^{\|\cdot\|_{B}}$ denotes the completion of $A$ in $B$. Also, we consider the notation for the applications $a_{0}: \mathbf{V} \times \mathbf{V} \rightarrow \mathbb{R}$, $a: H^{1}(\Omega) \times H^{1}(\Omega) \rightarrow \mathbb{R}, b_{0}: \mathbf{V} \times \mathbf{V} \times \mathbf{V} \rightarrow \mathbb{R}$, and $b: \mathbf{V} \times H^{1}(\Omega) \times H^{1}(\Omega) \rightarrow \mathbb{R}$, which are defined as

$$
\begin{aligned}
a_{0}(\mathbf{u}, \mathbf{v}) & =(\nabla \mathbf{u}, \nabla \mathbf{v}), \\
a(\phi, \psi) & =(\nabla \phi, \nabla \psi), \\
b_{0}(\mathbf{u}, \mathbf{v}, \mathbf{w}) & =((\mathbf{u} \cdot \nabla) \mathbf{v}, \mathbf{w}), \\
b(\mathbf{u}, \phi, \psi) & =(\mathbf{u} \cdot \nabla \phi, \psi),
\end{aligned}
$$

where $(\cdot, \cdot)$ is the standard inner product in $L^{2}(\Omega)$ or $\mathbf{L}^{2}(\Omega)$. It is well known that $a_{0}$ and $a$ are bilinear coercive forms and $b_{0}$ and $b$ are well defined trilinear forms with the following properties:

$$
\begin{aligned}
& b_{0}(\mathbf{u}, \mathbf{v}, \mathbf{w})=-b_{0}(\mathbf{u}, \mathbf{w}, \mathbf{v}) \\
& b(\mathbf{u}, \phi, \psi)=-b(\mathbf{u}, \psi, \phi), \\
& b_{0}(\mathbf{u}, \mathbf{v}, \mathbf{v})=0 \\
& b(\mathbf{u}, \phi, \phi)=0
\end{aligned}
$$

for all $\mathbf{u}, \mathbf{v}, \mathbf{w} \in \mathbf{V}$ and $\psi, \phi \in H^{1}(\Omega)$. Moreover, we need to introduce some notation related to some useful Sobolev inequalities and estimates for $b$ and $b_{0}$. There exist $C_{\text {poi }}>0$, $C_{\text {tr }}>0$, and $C_{1}$ depending only on $\Omega$ such that

$$
\begin{aligned}
\|\mathbf{u}\|_{\mathbf{L}^{2}(\Omega)} & \leq C_{\text {poi }}\|\mathbf{u}\|_{\mathbf{V}}, \\
\|c\|_{L^{2}(\Omega)} & \leq C_{\text {poi }}\|c\|_{\widetilde{H}^{1}(\Omega)}, \\
\|\varphi\|_{L^{1}(\partial \Omega)} & \leq C_{\text {tr }}\|\varphi\|_{W^{1,1}(\Omega)}, \\
\left|b_{0}(\mathbf{u}, \mathbf{v}, \mathbf{w})\right| & \leq C_{1}\|\mathbf{u}\|_{\mathbf{V}}\|\mathbf{v}\|_{\mathbf{V}}\|\mathbf{w}\|_{\mathbf{V}}, \\
|b(\mathbf{u}, c, n)| & \leq C_{1}\|\mathbf{u}\|_{\mathbf{V}}\|c\|_{\widetilde{H}^{1}(\Omega)}\|n\|_{\widetilde{H}^{1}(\Omega)},
\end{aligned}
$$

for all $\mathbf{u}, \mathbf{v}, \mathbf{w} \in \mathbf{V}, c, n \in \widetilde{H}^{1}(\Omega)$, and $\varphi \in W^{1,1}(\Omega)$. For details on Poincaré and trace inequalities, we refer to [8] and for the estimates of $b_{0}$ and $b$ consult [11].

The main result of the paper is the existence and uniqueness of weak solutions for (1)-(6). Indeed, let us introduce some appropriate notation:

$$
\begin{aligned}
\Theta_{1} & :=\frac{1-C_{\mathrm{tr}}}{1-C_{\mathrm{tr}}-2 \chi\|r\|_{L^{1}(\mathbb{R})} C_{\mathrm{tr}} C_{\mathrm{poi}}}, \\
\Theta_{2} & :=\frac{1-C_{\mathrm{tr}}}{1-C_{\mathrm{tr}}-C_{\mathrm{tr}} C_{\mathrm{poi}}}, \\
\Gamma_{0} & =\frac{|\Omega| \Theta_{1} C_{\mathrm{poi}}}{|\Omega|-\chi \beta \alpha_{1}\|r\|_{L^{\infty}(\mathbb{R})}^{2} C_{\mathrm{poi}}^{2} \Theta_{1} \Theta_{2}}\left[\frac{\chi \alpha_{1}\|r\|_{L^{\infty}(\mathbb{R})}^{2} \Theta_{2}}{\delta|\Omega|}\left\|f_{c}\right\|_{L^{2}(\Omega)}\right. \\
& \left.+\left\|f_{n}\right\|_{L^{2}(\Omega)}\right], \\
\Gamma_{1} & =\frac{\gamma S_{c} g C_{\mathrm{poi}}}{S_{c}-C_{1} C_{\mathrm{poi}}\left(\gamma g \Gamma_{0}+\|\mathbf{F}\|_{\mathrm{L}^{2}(\Omega)}\right)}, \\
\Gamma_{2} & =\frac{1-C_{\mathrm{tr}}}{1-2\|r\|_{L^{1}(\mathbb{R})}\left(1-C_{\mathrm{tr}}+C_{\mathrm{tr}} C_{\mathrm{poi}}\right)}, \\
\Gamma_{3} & =\frac{1-C_{\mathrm{tr}}}{\delta\left(1-C_{\mathrm{tr}}-C_{\mathrm{tr}} C_{\mathrm{poi}}\right)-\left(C_{1}\right)^{3}\|r\|_{\mathrm{Lip}(\mathbb{R})} \Gamma_{0}},
\end{aligned}
$$

such that the result is precised as follows.

Theorem 1. Let us consider that $f_{c}, f_{b} \in L^{2}(\Omega), \mathbf{F} \in \mathbf{L}^{2}(\Omega)$ and $\bar{n}$, the average of $n$ on $\Omega$, are given. Also consider notations (12)-(15). If we assume that the following assumptions,

$$
\begin{gathered}
r \in L^{\infty}(\mathbb{R}) \cap L^{1}(\mathbb{R}), \\
1-C_{t r}>C_{t r} C_{p o i} \max \left\{2 \chi\|r\|_{L^{1}(\mathbb{R})}, 1\right\}, \\
1>\chi \beta \bar{n}\|r\|_{L^{\infty}(\mathbb{R})}^{2} C_{p o i}^{2} \Theta_{1} \Theta_{2},
\end{gathered}
$$


are satisfied, there is $(\mathbf{u}, p, n, c) \in \mathbf{V} \times H^{1}(\Omega) \times H^{1}(\Omega) \times H^{1}(\Omega)$ satisfying (1)-(6). Moreover, if we consider that additionally $r \in$ Lip $(\mathbb{R})$ and the following inequalities,

$$
\begin{aligned}
& S_{c}-C_{1} C_{p o i}\left(\gamma g \Gamma_{0}+\|\mathbf{F}\|_{L^{2}(\Omega)}\right)>0, \\
& \delta\left(1-C_{t r}-C_{t r} C_{p o i}\right)-\left(C_{1}\right)^{3}\|r\|_{L^{1}(\mathbb{R})} \Gamma_{0}>0, \\
& C_{1}\|r\|_{L i p(\mathbb{R})} \Gamma_{0}<1, \\
& \Pi=\Gamma_{1} \Gamma_{2}\left\{C_{1} \Gamma_{0}\right. \\
& \quad+\frac{\|r\|_{L^{\infty}(\mathbb{R})} C \Gamma_{3} \Theta_{2} C_{p o i}}{\delta\left(1-C_{1}\|r\|_{L i p(\mathbb{R})} \Gamma_{0}\right)}\left[\beta C_{p o i}\|r\|_{L^{\infty}(\mathbb{R})} \Gamma_{0}\right. \\
& \left.\left.\quad+\left\|f_{c}\right\|_{L^{2}(\Omega)}\right]\right\}<1,
\end{aligned}
$$

are satisfied, the weak solution is unique.

It should be noted that existence and uniqueness results are derived in $[12,13]$ for the bioconvection problem, when the concentration of oxygen is assumed to be constant. In the case of [12], the proof is based on the application of the Galerkin approximation and in [13] on the application of the Gossez theorem. Moreover, other related results are given in $[3,5]$. In particular, in [5], a well detailed discussion of some particular models derived from (1)-(6) is given.

\section{Proof of Theorem 1}

2.1. Variational Formulation. By standard arguments, the variational formulation of (1)-(6) is given by

Find $(\mathbf{u}, n, c) \in \mathbf{V} \times H^{1}(\Omega) \times H^{1}(\Omega)$ such that

$$
\begin{array}{r}
S_{c} a_{0}(\mathbf{u}, \mathbf{v})+b_{0}(\mathbf{u}, \mathbf{u}, \mathbf{v})=\gamma S_{c}(n \mathbf{g}, \mathbf{v})+(\mathbf{F}, \mathbf{v}), \\
\forall \mathbf{v} \in \mathbf{V}, \\
a(n, \phi)+b(\mathbf{u}, n, \phi)=\chi(n r(c) \nabla c, \nabla \phi)+\left(f_{n}, \phi\right), \\
\forall \phi \in H^{1}(\Omega), \\
\delta a(c, \varphi)+b(\mathbf{u}, c, \varphi) \\
=-\beta(r(c) n, \varphi)+\delta \int_{\partial \Omega_{U}} \nabla c \cdot v \varphi d S+\left(f_{c}, \varphi\right),
\end{array}
$$$$
\forall \varphi \in H^{1}(\Omega) .
$$

We notice that if $f_{c}=f_{n}=0$ and $\mathbf{u}_{0}$ is a solution of (1)-(2) with $n=0$, we have that $\left(\mathbf{u}_{0}, 0,0\right)$ is a solution of (19). However, $\left(\mathbf{u}_{0}, 0,0\right)$ does not describe the bioconvective flow problem and we need to study the variational problem when the total local concentration of bacteria and the total local concentration of oxygen are some given strictly positive constants, that is, $\int_{\Omega} n_{\alpha} d \mathbf{x}=\alpha_{1}>0$ and $\int_{\Omega} c_{\alpha} d \mathbf{x}=\alpha_{2}>0$.
Thus, by considering the change of variable $\widehat{n}_{\alpha}=n_{\alpha}-\alpha_{1}|\Omega|^{-1}$ and $\widehat{c}_{\boldsymbol{\alpha}}=c_{\boldsymbol{\alpha}}-\alpha_{2}|\Omega|^{-1}$, we can rewrite (19) as follows:

$$
\begin{aligned}
& \text { Given } \left.\left.\left.\left.\boldsymbol{\alpha}=\left(\alpha_{2}, \alpha_{2}\right) \in\right] 0,1\right] \times\right] 0,1\right] \text { find }\left(\mathbf{u}_{\alpha}, \widehat{n}_{\boldsymbol{\alpha}}, \widehat{c}_{\boldsymbol{\alpha}}\right) \\
& \in \mathbf{V} \times \widetilde{H}^{1}(\Omega) \times \widetilde{H}^{1}(\Omega): \\
& S_{c} a_{0}\left(\mathbf{u}_{\boldsymbol{\alpha}}, \mathbf{v}\right)+b_{0}\left(\mathbf{u}_{\boldsymbol{\alpha}}, \mathbf{u}_{\alpha}, \mathbf{v}\right)=\gamma S_{c}\left(\widehat{n}_{\boldsymbol{\alpha}} \mathbf{g}, \mathbf{v}\right)+(\mathbf{F}, \mathbf{v}), \\
& a\left(\widehat{n}_{\boldsymbol{\alpha}}, \phi\right)+b\left(\mathbf{u}_{\alpha}, \widehat{n}_{\boldsymbol{\alpha}}, \phi\right) \\
& \quad=\chi\left(\left(\widehat{n}_{\boldsymbol{\alpha}}+\frac{\alpha_{1}}{|\Omega|}\right) r\left(\widehat{c}_{\boldsymbol{\alpha}}+\frac{\alpha_{2}}{|\Omega|}\right) \nabla \widehat{c}_{\boldsymbol{\alpha}}, \nabla \phi\right) \\
& \quad+\left(f_{n}, \phi\right), \\
& \delta a\left(\widehat{c}_{\boldsymbol{\alpha}}, \varphi\right)+b\left(\mathbf{u}_{\boldsymbol{\alpha}}, \widehat{c}_{\boldsymbol{\alpha}}, \varphi\right) \\
& \quad=-\beta\left(r\left(\widehat{c}_{\boldsymbol{\alpha}}+\frac{\alpha_{2}}{|\Omega|}\right)\left(\widehat{n}_{\boldsymbol{\alpha}}+\frac{\alpha_{1}}{|\Omega|}\right), \varphi\right) \\
& \quad+\delta \int_{\partial \Omega_{U}} \nabla \widehat{c}_{\boldsymbol{\alpha}} \cdot \boldsymbol{\nu} \varphi d S+\left(f_{c}, \varphi\right), \\
& \quad \forall(\mathbf{v}, \phi, \varphi) \in \mathbf{V} \times \widetilde{H}^{1}(\Omega) \times \widetilde{H}^{1}(\Omega) .
\end{aligned}
$$

\subsection{Some A Priori Estimates for $\mathbf{u}_{\alpha}, \widehat{n}_{\boldsymbol{\alpha}}$, and $\widehat{c}_{\boldsymbol{\alpha}}$}

Proposition 2. Consider that the assumptions for the existence result of Theorem 1 are satisfied. If we assume that $\left(\mathbf{u}_{\alpha}, \widehat{n}_{\alpha}, \widehat{c}_{\alpha}\right)$ is a solution of (20)-(24), then $\left\|\widehat{n}_{\alpha}\right\|_{\widetilde{H}^{1}(\Omega)} \leq \Gamma_{0}$ with $\Gamma_{0}$ defined on (13). Furthermore, the following estimates are valid:

$$
\begin{aligned}
\left\|\mathbf{u}_{\alpha}\right\|_{\mathrm{V}} & \leq C_{p o i}\left(\gamma g \Gamma_{0}+\|\mathbf{F}\|_{\mathbf{L}^{2}(\Omega)}\right), \\
\left\|\widehat{c}_{\alpha}\right\|_{\widetilde{H}^{1}(\Omega)} & \leq \frac{\Theta_{2} C_{p o i}}{\delta}\left[\beta C_{p o i}\|r\|_{L^{\infty}(\mathbb{R})} \Gamma_{0}+\left\|f_{c}\right\|_{L^{2}(\Omega)}\right] .
\end{aligned}
$$

Proof. In order to prove the estimates, we select the test functions $(\mathbf{v}, \phi, \varphi)=\left(\mathbf{u}_{\boldsymbol{\alpha}}, \widehat{n}_{\boldsymbol{\alpha}}, \widehat{c}_{\boldsymbol{\alpha}}\right)$ in (21)-(23). From (21) and (10), we deduce that

$$
\left\|\mathbf{u}_{\boldsymbol{\alpha}}\right\|_{\mathbf{V}} \leq \gamma g C_{\mathrm{poi}}^{2}\left\|\widehat{n}_{\boldsymbol{\alpha}}\right\|_{\widetilde{H}^{1}(\Omega)}+\left(S_{c}\right)^{-1} C_{\mathrm{poi}}\|\mathbf{F}\|_{\mathbf{L}^{2}(\Omega)} .
$$

Now, by the trace inequality and integration by parts, we have that

$$
\begin{aligned}
\int_{\partial \Omega}\left|\nabla \widehat{n}_{\boldsymbol{\alpha}} \cdot v \widehat{n}_{\boldsymbol{\alpha}}\right| d S \leq & C_{\text {tr }}\left\|\widehat{n}_{\boldsymbol{\alpha}} \nabla \widehat{n}_{\boldsymbol{\alpha}} \cdot \nu\right\|_{W^{1,1}(\Omega)} \\
\leq & C_{\text {tr }} C_{\text {poi }}\left\|\widehat{n}_{\boldsymbol{\alpha}}\right\|_{\widetilde{H}^{1}(\Omega)}^{2} \\
& +C_{\text {tr }} \int_{\partial \Omega}\left|\nabla \widehat{n}_{\boldsymbol{\alpha}} \cdot \nu \widehat{n}_{\boldsymbol{\alpha}}\right| d S
\end{aligned}
$$

which implies that

$$
\int_{\partial \Omega}\left|\nabla \widehat{n}_{\boldsymbol{\alpha}} \cdot \nu \widehat{n}_{\boldsymbol{\alpha}}\right| d S \leq \frac{C_{\mathrm{tr}} C_{\mathrm{poi}}}{1-C_{\mathrm{tr}}}\left\|\widehat{n}_{\boldsymbol{\alpha}}\right\|_{\widetilde{H}^{1}(\Omega)}^{2} .
$$


Here, we have used the fact that $1-C_{\mathrm{tr}}>0$, as a consequence of the assumption (16). Then, by integration by parts we get the bound

$$
\begin{aligned}
\left(\widehat{n}_{\boldsymbol{\alpha}} r\left(\widehat{c}_{\boldsymbol{\alpha}}+\frac{\alpha_{2}}{|\Omega|}\right) \nabla \widehat{c}_{\boldsymbol{\alpha}}, \nabla \widehat{n}_{\boldsymbol{\alpha}}\right) \\
=\left(\nabla\left[\int_{0}^{\widehat{c}_{\boldsymbol{\alpha}}} r\left(m+\frac{\alpha_{2}}{|\Omega|}\right) d m\right], \nabla\left(\frac{\widehat{n}_{\boldsymbol{\alpha}}^{2}}{2}\right)\right) \\
=-\left(\int_{0}^{\widehat{c}_{\boldsymbol{\alpha}}} r\left(m+\frac{\alpha_{2}}{|\Omega|}\right) d m, \Delta\left(\frac{\widehat{n}_{\boldsymbol{\alpha}}}{2}\right)\right) \\
\quad+\int_{\partial \Omega}\left[\int_{0}^{\widehat{c}_{\boldsymbol{\alpha}}} r\left(m+\frac{\alpha_{2}}{|\Omega|}\right) d m\right] \nabla\left(\frac{\widehat{n}_{\boldsymbol{\alpha}}^{2}}{2}\right) \cdot \nu d S \\
\leq 2\|r\|_{L^{1}(\mathbb{R})} \int_{\partial \Omega}\left|\widehat{n}_{\boldsymbol{\alpha}} \nabla \widehat{n}_{\boldsymbol{\alpha}} \cdot \nu\right| d S \\
\leq \frac{2\|r\|_{L^{1}(\mathbb{R})} C_{\mathrm{tr}} C_{\mathrm{poi}}\left\|\widehat{n}_{\boldsymbol{\alpha}}\right\|_{\widetilde{H}^{1}(\Omega)}^{2} .}{1-C_{\mathrm{tr}}}
\end{aligned}
$$

From (22), using the properties (10) and the inequality (29), we have that

$$
\begin{aligned}
\left\|\widehat{n}_{\boldsymbol{\alpha}}\right\|_{\widetilde{H}^{1}(\Omega)}^{2}= & \chi\left(\widehat{n}_{\boldsymbol{\alpha}} r\left(\widehat{c}_{\boldsymbol{\alpha}}+\frac{\alpha_{2}}{|\Omega|}\right) \nabla \widehat{c}_{\boldsymbol{\alpha}}, \nabla \widehat{n}_{\boldsymbol{\alpha}}\right) \\
& +\frac{\chi \alpha_{1}}{|\Omega|}\left(r\left(\widehat{c}_{\boldsymbol{\alpha}}+\frac{\alpha_{2}}{|\Omega|}\right) \nabla \widehat{c}_{\boldsymbol{\alpha}}, \nabla \widehat{n}_{\boldsymbol{\alpha}}\right) \\
& +\left(f_{n}, \phi\right) \\
\leq & \frac{2 \chi\|r\|_{L^{1}(\mathbb{R})} C_{\mathrm{tr}} C_{\mathrm{poi}}}{1-C_{\mathrm{tr}}}\left\|\widehat{n}_{\boldsymbol{\alpha}}\right\|_{\widetilde{H}^{1}(\Omega)}^{2} \\
& +\frac{\chi \alpha_{1}}{|\Omega|}\|r\|_{L^{\infty}(\mathbb{R})}\left\|\widehat{c}_{\boldsymbol{\alpha}}\right\|_{\widetilde{H}^{1}(\Omega)}\left\|\widehat{n}_{\boldsymbol{\alpha}}\right\|_{\widetilde{H}^{1}(\Omega)} \\
& +C_{\text {poi }}\left\|f_{n}\right\|_{L^{2}(\Omega)}\left\|\widehat{n}_{\boldsymbol{\alpha}}\right\|_{\widetilde{H}^{1}(\Omega)},
\end{aligned}
$$

or equivalently, we get the following estimate for $\widehat{n}_{\alpha}$ :

$$
\begin{aligned}
& \left\|\widehat{n}_{\boldsymbol{\alpha}}\right\|_{\widetilde{H}^{1}(\Omega)} \\
& \quad \leq \Theta_{1}\left[\frac{\chi \alpha_{1}}{|\Omega|}\|r\|_{L^{\infty}(\mathbb{R})}\left\|\widehat{c}_{\boldsymbol{\alpha}}\right\|_{\widetilde{H}^{1}(\Omega)}+C_{\text {poi }}\left\|f_{n}\right\|_{L^{2}(\Omega)}\right],
\end{aligned}
$$

with $\Theta_{1}$ being defined in (12). Similarly, from (23) and (28) with $\widehat{c}_{\boldsymbol{\alpha}}$ instead of $\widehat{n}_{\boldsymbol{\alpha}}$, we deduce that

$\left\|\widehat{c}_{\boldsymbol{\alpha}}\right\|_{\widetilde{H}^{1}(\Omega)}$

$$
\leq \frac{\Theta_{2} C_{\text {poi }}}{\delta}\left[\beta C_{\text {poi }}\|r\|_{L^{\infty}(\mathbb{R})}\left\|\widehat{n}_{\boldsymbol{\alpha}}\right\|_{\widetilde{H}^{1}(\Omega)}+\left\|f_{c}\right\|_{L^{2}(\Omega)}\right],
$$

where $\Theta_{2}$ is given in (12). Now, replacing the estimate (32) in (31) and applying (16), we deduce the existence of $\Gamma_{0}$ defined in (13) such that $\left\|\widehat{n}_{\boldsymbol{\alpha}}\right\|_{\widetilde{H}^{1}(\Omega)} \leq \Gamma_{0}$. We notice that the second and third relation in (16) imply that $\Theta_{i}>1, i=1,2$, and $|\Omega|>$ $\chi \beta \alpha_{1}\|r\|_{L^{\infty}(\mathbb{R})}^{2} C_{\text {poi }}^{2} \Theta_{1} \Theta_{2}$, respectively, that is, $\Gamma>0$ under (16). Moreover, from (26) and (31), we deduce the estimates given in (25) for $\left\|\mathbf{u}_{\boldsymbol{\alpha}}\right\|_{\mathbf{V}}$ and $\left\|\widehat{c}_{\boldsymbol{\alpha}}\right\|_{\widetilde{H}^{1}(\Omega)}$, concluding the proof of the Proposition.
2.3. Proof of Theorem 1. To prove the existence, we can apply the Gossez theorem $[9,14]$. Let us first define the mapping $G: \mathbf{V} \times \widetilde{H}^{1}(\Omega) \times \widetilde{H}^{1}(\Omega) \rightarrow\left(\mathbf{V} \times \widetilde{H}^{1}(\Omega) \times \widetilde{H}^{1}(\Omega)\right)^{\prime}$ by the relation

$$
\begin{aligned}
& \ll G(\mathbf{u}, n, c),(\mathbf{v}, \phi, \varphi) 》=\lambda_{1}\left\{S_{c} a_{0}(\mathbf{u}, \mathbf{v})+b_{0}(\mathbf{u}, \mathbf{u}, \mathbf{v})\right. \\
& \left.-\gamma S_{c}(n \mathbf{g}, \mathbf{v})-(\mathbf{F}, \mathbf{v})\right\}+\lambda_{2}\{a(n, \phi)+b(\mathbf{u}, n, \phi) \\
& \left.-\chi\left(\left(n+\frac{\alpha_{1}}{|\Omega|}\right) r\left(c+\frac{\alpha_{2}}{|\Omega|}\right) \nabla c, \nabla \phi\right)-\left(f_{n}, \phi\right)\right\} \\
& +\lambda_{3}\{\delta a(c, \varphi)+b(\mathbf{u}, c, \varphi) \\
& +\beta\left(r\left(c+\frac{\alpha_{2}}{|\Omega|}\right)\left(n+\frac{\alpha_{1}}{|\Omega|}\right), \varphi\right)-\delta \int_{\partial \Omega_{U}} \nabla c \\
& \left.\cdot \nu \varphi d S-\left(f_{c}, \varphi\right)\right\}, \\
& \forall(\mathbf{u}, n, c),(\mathbf{v}, \phi, \varphi) \in \mathbf{V} \times \widetilde{H}^{1}(\Omega) \times \widetilde{H}^{1}(\Omega),
\end{aligned}
$$

with $\langle\cdot \cdot, \cdot\rangle$ denoting the duality pairing between $\mathbf{V} \times \widetilde{H}^{1}(\Omega) \times$ $\widetilde{H}^{1}(\Omega)$ and $\left(\mathbf{V} \times \widetilde{H}^{1}(\Omega) \times \widetilde{H}^{1}(\Omega)\right)^{\prime}$ and $\lambda_{1}, \lambda_{2}$, and $\lambda_{3}$ are positive fixed constant. From (10), (12), and (29), we then have that

$$
\begin{aligned}
& \ll G(\mathbf{u}, n, c),(\mathbf{u}, n, c) 》 \geq\left\{\lambda_{1} S_{c}\|\mathbf{u}\|_{\mathbf{V}}^{2}\right. \\
& \left.-\lambda_{1} \gamma S_{c} g\left(C_{\text {poi }}\right)^{2}\|n\|_{\widetilde{H}^{1}(\Omega)}\|\mathbf{u}\|_{\mathbf{V}}+\frac{\lambda_{2}}{3 \Theta_{1}}\|n\|_{\widetilde{H}^{1}(\Omega)}^{2}\right\} \\
& +\left\{\frac{\lambda_{2}}{3 \Theta_{1}}\|n\|_{\widetilde{H}^{1}(\Omega)}^{2}\right. \\
& -\frac{\lambda_{2} \chi \alpha_{1}}{|\Omega|}\|r\|_{L^{\infty}(\mathbb{R})}\|c\|_{\widetilde{H}^{1}(\Omega)}\|n\|_{\widetilde{H}^{1}(\Omega)} \\
& \left.+\frac{\lambda_{3} \delta}{2 \Theta_{2}}\|c\|_{\widetilde{H}^{1}(\Omega)}^{2}\right\}+\left\{\frac{\lambda_{3} \delta}{2 \Theta_{2}}\|c\|_{\widetilde{H}^{1}(\Omega)}^{2}\right. \\
& -\lambda_{3} \beta\left(C_{\text {poi }}\right)^{2}\|r\|_{L^{\infty}(\mathbb{R})}\|c\|_{\widetilde{H}^{1}(\Omega)}\|n\|_{\widetilde{H}^{1}(\Omega)} \\
& \left.+\frac{\lambda_{2}}{3 \Theta_{1}}\|n\|_{\widetilde{H}^{1}(\Omega)}^{2}\right\}-C_{\text {poi }}\left\{\lambda_{1}\|\mathbf{F}\|_{L^{2}(\Omega)}\|\mathbf{u}\|_{\mathbf{V}}\right. \\
& \left.+\lambda_{2}\left\|f_{n}\right\|_{L^{2}(\Omega)}\|n\|_{\widetilde{H}^{1}(\Omega)}+\lambda_{3}\left\|f_{c}\right\|_{L^{2}(\Omega)}\|c\|_{\widetilde{H}^{1}(\Omega)}\right\} \\
& :=\Upsilon_{1}+\Upsilon_{2}-\Upsilon_{3} .
\end{aligned}
$$

Now, selecting $\lambda_{1}, \lambda_{2}, \lambda_{3}$ and $r$ such that

$$
\begin{aligned}
& \lambda_{1}<\frac{4 \lambda_{2}}{3 \Theta_{1} \gamma^{2} g^{2} S_{c}\left(C_{\text {poi }}\right)^{4}}, \\
& \lambda_{2}<\frac{4 \delta|\Omega|^{2} \lambda_{3}}{6 \Theta_{1} \Theta_{2}\left(\chi \alpha_{1}\|r\|_{L^{\infty}(\mathbb{R})}\right)^{2}},
\end{aligned}
$$




$$
\begin{aligned}
& \lambda_{3}<\frac{4 \delta \lambda_{2}}{6 \Theta_{1} \Theta_{2}\left(\beta\left(C_{\text {poi }}\right)^{2}\|r\|_{L^{\infty}(\mathbb{R})}\right)^{2}} \\
& r<\frac{\Upsilon_{1}+\Upsilon_{2}}{C_{\text {poi }}\left(\lambda_{1}\|\mathbf{F}\|_{L^{2}(\Omega)}+\lambda_{2}\left\|f_{n}\right\|_{L^{2}(\Omega)}+\lambda_{3}\left\|f_{c}\right\|_{L^{2}(\Omega)}\right)},
\end{aligned}
$$

we can prove that $\langle\langle G(\mathbf{u}, n, c),(\mathbf{u}, n, c)\rangle$ is positive for all $(\mathbf{u}, n, c) \in \mathbf{V} \times \widetilde{H}^{1}(\Omega) \times \widetilde{H}^{1}(\Omega)$ such that $\|(\mathbf{u}, n$, c) $\|_{\mathbf{V} \times \widetilde{H}^{1}(\Omega) \times \widetilde{H}^{1}(\Omega)}=r$. Moreover, we notice that it is straightforward to deduce that $G$ is continuous between the weak topologies of $\mathbf{V} \times \widetilde{H}^{1}(\Omega) \times \widetilde{H}^{1}(\Omega)$ and $\left(\mathbf{V} \times \widetilde{H}^{1}(\Omega) \times \widetilde{H}^{1}(\Omega)\right)^{\prime}$. Thus, there is $(\mathbf{u}, n, c) \in \bar{B}_{r}(0) \subset \mathbf{V} \times \widetilde{H}^{1}(\Omega) \times \widetilde{H}^{1}(\Omega)$ such that $\langle G(\mathbf{u}, n, c),(\mathbf{u}, n, c)\rangle=0$, concluding the proof of existence.

To prove the uniqueness we consider that there are two solutions $\left(\mathbf{u}^{i}, n^{i}, c^{i}\right), i=1,2$, satisfying (21)-(23). Then, subtracting, selecting the test functions $(\mathbf{v}, \phi, \varphi)=\left(\mathbf{u}^{1}-\mathbf{u}^{2}, n^{1}-\right.$ $\left.n^{2}, c^{1}-c^{2}\right)$, using (10), (16), (17), and applying Proposition 2, we get

$$
\begin{aligned}
& \left\|\mathbf{u}^{1}-\mathbf{u}^{2}\right\|_{\mathbf{V}} \leq \Gamma_{1}\left\|n^{1}-n^{2}\right\|_{\widetilde{H}^{1}(\Omega)}, \\
& \left\|n^{1}-n^{2}\right\|_{\widetilde{H}^{1}(\Omega)} \leq \Gamma_{2}\left[C_{1}\left\|\mathbf{u}^{1}-\mathbf{u}^{2}\right\|_{\mathbf{V}}\left\|n^{1}\right\|_{\widetilde{H}^{1}(\Omega)}\right. \\
& \left.\quad+\|r\|_{L^{\infty}(\mathbb{R})}\left\|c^{1}-c^{2}\right\|_{\widetilde{H}^{1}(\Omega)}\right], \\
& \left\|c^{1}-c^{2}\right\|_{\widetilde{H}^{1}(\Omega)} \leq C_{1} \Gamma_{3}\left[\left\|\mathbf{u}^{1}-\mathbf{u}^{2}\right\|_{\mathbf{V}}\left\|c^{2}\right\|_{\widetilde{H}^{1}(\Omega)}\right. \\
& \left.\quad+\left(C_{1}\right)^{2}\|r\|_{\operatorname{Lip}(\mathbb{R})}\left\|n^{1}\right\|_{\widetilde{H}^{1}(\Omega)}\left\|c^{1}-c^{2}\right\|_{\widetilde{H}^{1}(\Omega)}\right],
\end{aligned}
$$

with $\Gamma_{i}$ being defined in (13)-(15). From (38), Proposition 2, and the first inequality in (18), we have that

$$
\begin{aligned}
& \left\|c^{1}-c^{2}\right\|_{\widetilde{H}^{1}(\Omega)} \\
& \leq \frac{C_{1} \Gamma_{3} \Theta_{2} C_{\text {poi }}}{\delta\left(1-\left(C_{1}\right)^{2}\|r\|_{\operatorname{Lip}(\mathbb{R})} \Gamma_{0}\right)}\left[\beta C_{\text {poi }}\|r\|_{L^{\infty}(\mathbb{R})} \Gamma_{0}\right. \\
& \left.\quad+\left\|f_{c}\right\|_{L^{2}(\Omega)}\right]\left\|\mathbf{u}^{1}-\mathbf{u}^{2}\right\|_{\mathbf{V}} .
\end{aligned}
$$

Then, replacing (39) in (37), using Proposition 2 to estimate $\left\|n^{1}\right\|_{\widetilde{H}^{1}(\Omega)}$, we obtain the bound $\left\|n^{1}-n^{2}\right\|_{\widetilde{H}^{1}(\Omega)} \leq \Pi\left(\Gamma_{1}\right)^{-1} \| \mathbf{u}^{1}-$ $\mathbf{u}^{2} \|_{\mathbf{V}}$ with $\Pi$ being defined in (18). Now, using this estimate in (36), we get that $\left\|\mathbf{u}^{1}-\mathbf{u}^{2}\right\|_{\mathbf{V}} \leq \Pi\left\|\mathbf{u}^{1}-\mathbf{u}^{2}\right\|_{\mathbf{V}}$. Thus using the fact that $\Pi \leq 1$ we deduce that $\mathbf{u}^{1}=\mathbf{u}^{2}$ on $\mathbf{V}$, which also implies that $n^{1}=n^{2}$ and $c^{1}=c^{2}$ on $\widetilde{H}^{1}(\Omega)$, concluding the uniqueness proof.

\section{Conflicts of Interest}

The authors declare that they have no conflicts of interest.

\section{Authors' Contributions}

All authors contributed equally to the writing of this paper. All authors read and approved the final manuscript.

\section{Acknowledgments}

The authors are supported by the project DIUBB 172409 GI/C at Universidad del Bío-Bío, Chile. Aníbal Coronel is supported by the projects DIUBB 183309 4/R and FAPEI at U. del Bío-Bío, Chile. Alex Tello and Ian Hess are supported by Conicyt-Chile through the grants program "Becas de Doctorado".

\section{References}

[1] Y. Moribe, On the bioconvection of Tetrahymena pyriformis [M.S. thesis], Osaka University, (Japanese) 1973.

[2] M. Levandowsky, W. S. Childress, S. H. Hutner, and E. A. Spiegel, "A mathematical model of pattern formation by swimming microorganisms," The Journal of Protozoology, vol. 22, no. 2, pp. 296-306, 1975.

[3] Y. Kan-on, K. Narukawa, and Y. Teramoto, "On the equations of bioconvective flow," Journal of Mathematics of Kyoto University, vol. 32, no. 1, pp. 135-153, 1992.

[4] I. Tuval, L. Cisneros, Ch. Dombrowski, C. W. Wolgemuth, J. O. Kessler, and R. E. Goldstein, "Bacterial swimming and oxygen transport near contact lines," Proceedings of the National Acadamy of Sciences of the United States of America, vol. 102, no. 7, pp. 2277-2282, 2005.

[5] J.-G. Liu and A. Lorz, "A coupled chemotaxis-fluid model: global existence," Annales de l'Institut Henri Poincaré. Analyse Non Linéaire, vol. 28, no. 5, pp. 643-652, 2011.

[6] H. G. Lee and J. Kim, "Numerical investigation of falling bacterial plumes caused by bioconvection in a three-dimensional chamber," European Journal of Mechanics B/Fluids, vol. 52, pp. 120-130, 2015.

[7] R. A. Adams, Sobolev Spaces, Academic Press, New York, NY, USA, 1975.

[8] F. Boyer and P. Fabrie, Mathematical Tools for the Study of the Incompressible Navier-Stokes Equations and Related Models, vol. 183 of Applied Mathematical Sciences, Springer, New York, NY, USA, 2013.

[9] H. I. Ene and D. Poliševski, Thermal Flows in Porous Media, Kluwer Academic Publishers, Dordrecht, the Netherlands, 1987.

[10] O. A. Ladyzhenskaya, The Mathematical Theory of Viscous Incompressible Flow, Gordon and Breach, New York, NY, USA, 1969.

[11] R. Temam, Navier-Stokes Equations. Theory and Numerical Analysis, vol. 2 of Studies in Mathematics and Its Applications, North-Holland Publishing Co., Amsterdam, the Netherlands, 1977.

[12] J. L. Boldrini, M. A. Rojas-Medar, and M. D. Rojas-Medar, "Existence and uniqueness of stationary solutions to bioconvective flow equations," Electronic Journal of Differential Equations, no. 110, 15 pages, 2013.

[13] A. Cǎpăţînǎ and R. Stavre, "A control problem in biconvective flow," Journal of Mathematics of Kyoto University, vol. 37, no. 4, pp. 585-595, 1997.

[14] J.-P. Gossez, "Remarques sur les opérateurs monotones," Académie Royale de Belgique. Bulletin de la Classe des Sciences, vol. 52, no. 5, pp. 1073-1077, 1966 (French). 


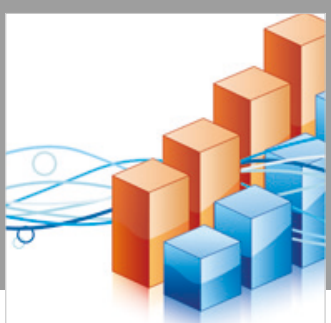

Advances in

Operations Research

\section{-n-m}
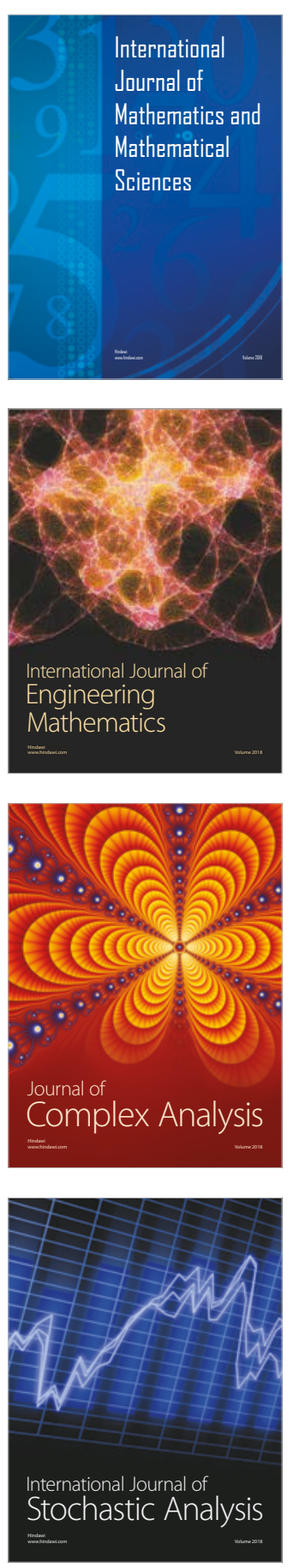
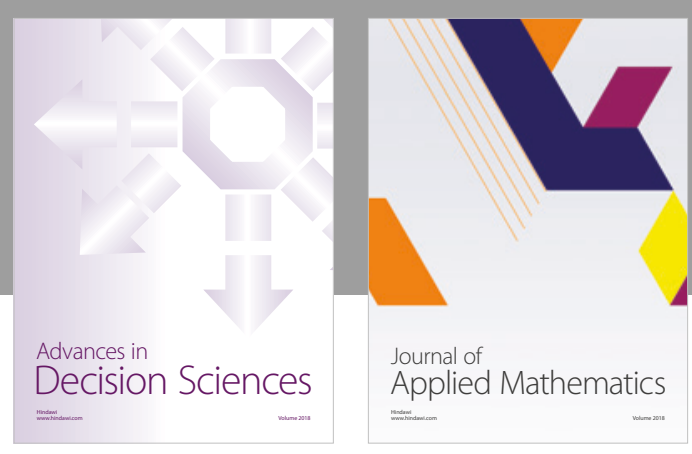

Journal of

Applied Mathematics
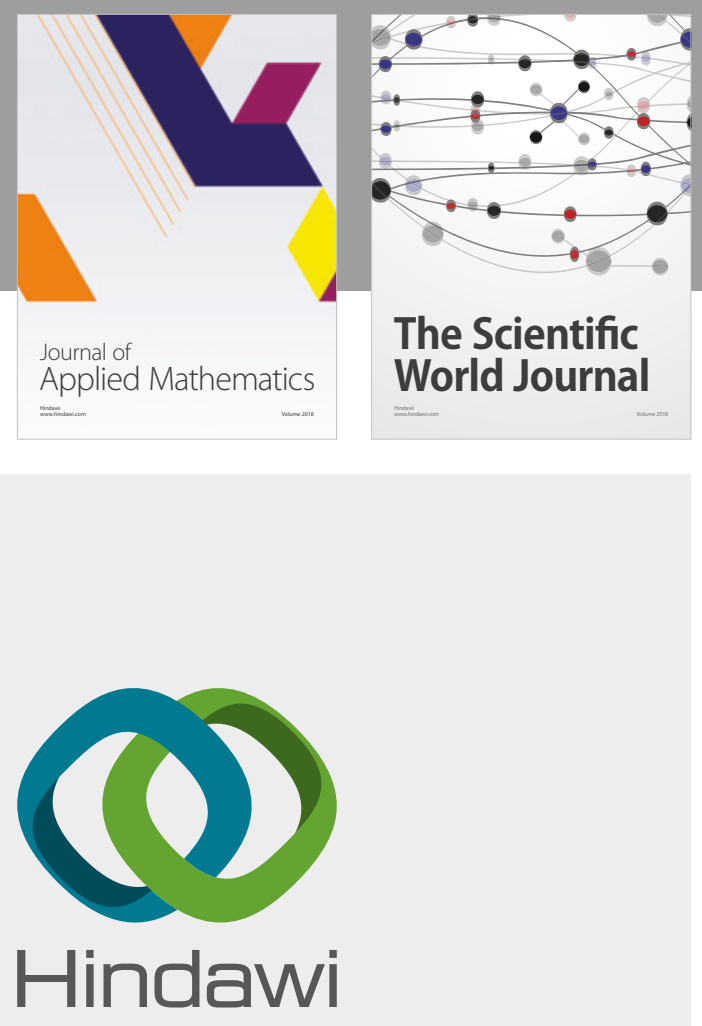

Submit your manuscripts at

www.hindawi.com

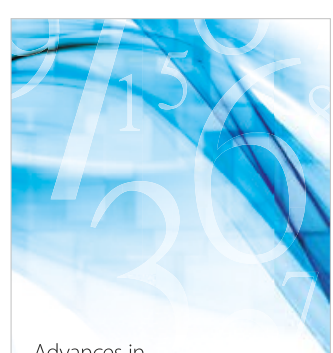

Advances in
Numerical Analysis
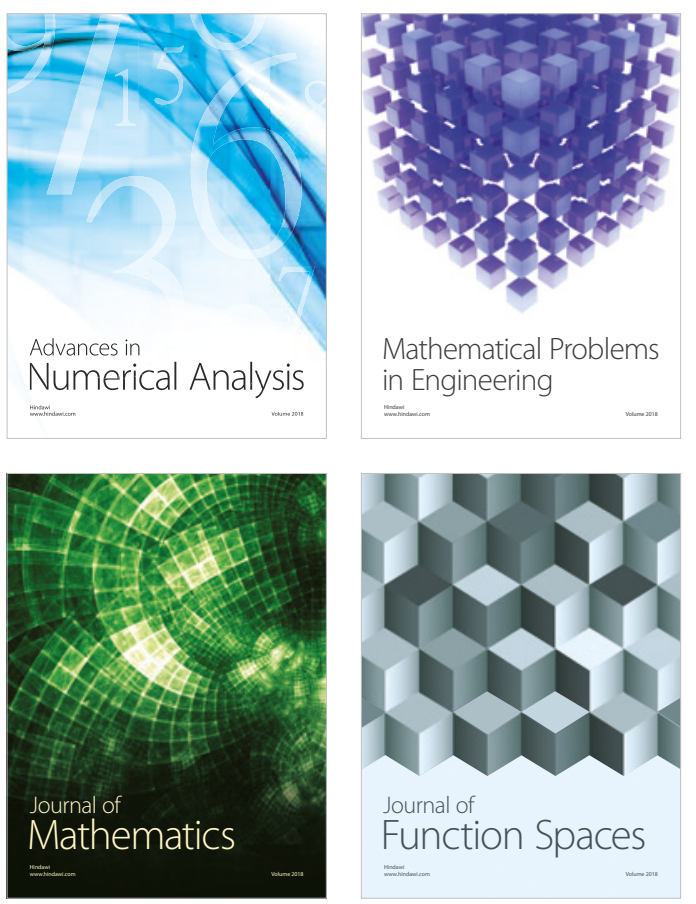

Mathematical Problems in Engineering

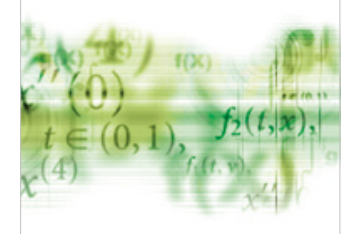

International Journal of

Differential Equations

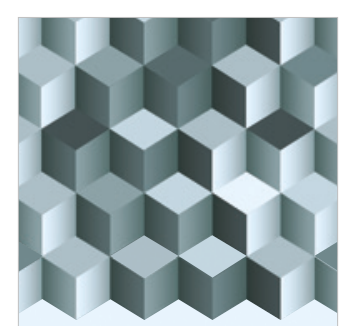

Journal of

Function Spaces

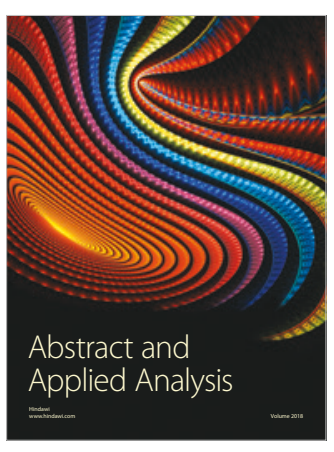

The Scientific

World Journal

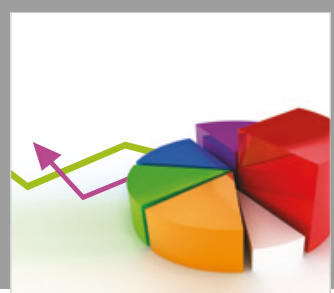

Journal of

Probability and Statistics
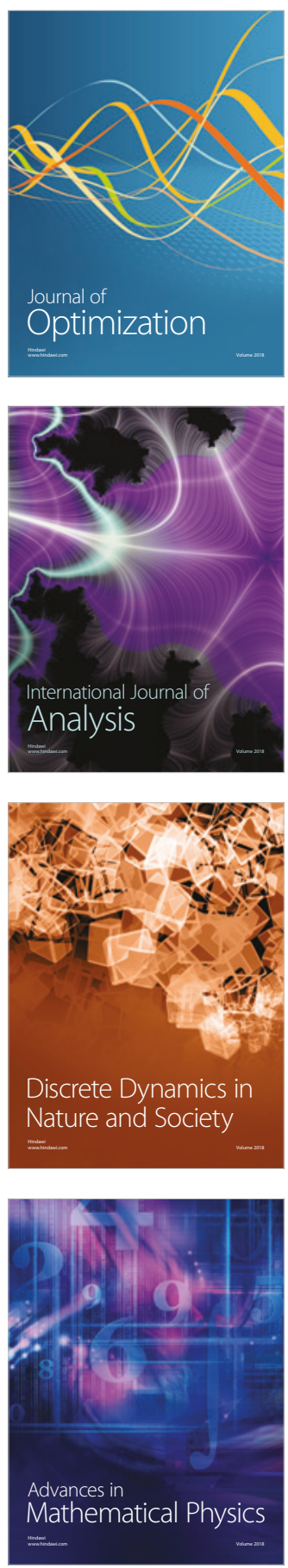